\title{
Flavour-violating decays of mixed top-charm squarks at the LHC
}

\author{
Amit Chakraborty $^{1, \mathrm{a}}{ }_{\mathbb{C}}$, Motoi Endo ${ }^{1, \mathrm{~b}}$, Benjamin Fuks ${ }^{2,3, \mathrm{c}}$, Björn Herrmann $^{4, \mathrm{~d}}$, Mihoko M. Nojiri ${ }^{1,5,6, \mathrm{e}}$, \\ Priscilla Pani $^{7, f}$, Giacomo Polesello ${ }^{8, g}$ \\ ${ }^{1}$ Theory Center, IPNS, KEK, Tsukuba, Ibaraki 305-0801, Japan \\ 2 Sorbonne Université, CNRS, Laboratoire de Physique Théorique et Hautes Energies, LPTHE, 75005 Paris, France \\ ${ }^{3}$ Institut Universitaire de France, 103 boulevard Saint-Michel, 75005 Paris, France \\ ${ }^{4}$ Univ. Grenoble Alpes, USMB, CNRS, LAPTh, 74000 Annecy, France \\ 5 The Graduate University of Advanced Studies (Sokendai), Tsukuba, Ibaraki 305-0801, Japan \\ ${ }^{6}$ Kavli IPMU (WPI), University of Tokyo, Kashiwa, Chiba 277-8583, Japan \\ ${ }^{7}$ CERN, Experimental Physics Department, 1211 Geneva 23, Switzerland \\ ${ }^{8}$ INFN, Sezione di Pavia, Via Bassi 6, 27100 Pavia, Italy
}

Received: 28 August 2018 / Accepted: 10 October 2018 / Published online: 20 October 2018

(C) The Author(s) 2018

\begin{abstract}
We explore signatures related to squark decays in the framework of non-minimally flavour-violating supersymmetry. We consider a simplified model where the lightest squark consists of an admixture of charm and top flavour. By recasting the existing LHC searches for top and charm squarks, we show that the limits on squark masses from these analyses are significantly weakened when the topcharm mixing is sizeable. We propose a dedicated search for squarks based on the $t c+E_{\mathrm{T}}^{\text {miss }}$ final state which enhances the experimental sensitivity for the case of high mixing, and we map its expected reach for the forthcoming runs of the LHC. We emphasize the role of analyses requiring a jet tagged as produced by the fragmentation of a charm quark in understanding the squark mixing pattern, thus providing a novel handle on new physics. Our results show that, in order to achieve full coverage of the parameter space of supersymmetric models, it is necessary to extend current experimental search programmes with analyses specifically targeting the cases where the lightest top-partner is a mixed state.
\end{abstract}

\section{Introduction}

While the Large Hadron Collider (LHC) pursues its quest for new physics, its Run 2 at a centre-of-momentum energy

\footnotetext{
a e-mail: amit@post.kek.jp

be-mail: motoi.endo@kek.jp

ce-mail: fuks@1pthe.jussieu.fr

d e-mail: herrmann@lapth.cnrs.fr

ee-mail: nojiri@post.kek.jp

f e-mail: priscilla.pani@cern.ch

g e-mail: giacomo.polesello@cern.ch
}

of $13 \mathrm{TeV}$ being on-going, no direct evidence for physics beyond the Standard Model (SM) has been observed so far. The conceptual problems and limitations of the Standard Model therefore remain unsolved. Among the various theoretical frameworks tackling those issues, Supersymmetry still remains one of the most popular and appealing options. The absence of experimental evidence for any of the superpartners of the Standard Model degrees of freedom, and in particular of the strongly-interacting squarks and gluinos [115], however imposes strong constraints on how Supersymmetry could be viably realised. For instance, the superpartners have to be too heavy to be produced at current LHC centre-of-mass energy and luminosity, or the particle spectrum has to be highly degenerate [16-18]. An alternative to these two solutions would be to abandon the idea of a minimal supersymmetric realisation which most current searches are based upon.

The ATLAS and CMS collaborations have obtained their limits under the assumption of a rather simplified realisation of the Minimal Supersymmetric Standard Model (MSSM). Those studies generally consider that a limited number of new physics states are light and can thus be produced at the LHC, and that those light particles therefore undergo a single well-defined decay mode. While studies of such simplified situations are reasonable and important starting points, the structure of the MSSM could be more general and complex and yield decay patterns that are not addressed by current searches.

In the present work, we address this last point and consider a realisation of the MSSM where inter-generational mixings in the squark sector are allowed. In this case, a physical squark eigenstate contains several components of 
well-defined flavour, which opens the door to multiple decay modes. For example, if the lightest squark is dominantly of top flavour but additionally contains a sizeable charm-flavour component, decays involving a top quark and a charm quark may both have a significant branching fraction. As a consequence, not all signatures stemming from squark pair production and decay are captured by the existing experimental searches, and current direct search limits may be weakened $[19,20]$.

In the MSSM realisations usually under consideration, squark inter-generational mixings are suppressed by imposing the minimal flavour violation (MFV) paradigm in which all flavour-violating interactions originate from the diagonalisation of the fermion sector and the corresponding CKM and PMNS matrices. Departing from an MFV squark sector, additional flavour-mixing terms may be present in the Lagrangian of the theory. By virtue of kaon data constraints, any mixing involving squarks of the first generation has to be extremely small [21], although second and third generation squark mixings are still largely allowed by current data [22]. We therefore focus on mixings solely involving the charm and top flavours of right-handed squarks. Such mixings can for instance originate from Grand Unification at a high scale $[23,24]$, and their TeV scale implications on squark production and decay have received considerable attention in the past [25-37]. In this work, we assess how they are constrained by the most recent LHC results and show, by proposing a novel class of search strategies, how future LHC searches could be tailored better to constrain non-minimally flavourviolating (NMFV) Supersymmetry.

The rest of this paper is organised as follows. In Sect. 2, we introduce the simplified setup that we have adopted to include inter-generational squark mixings in the MSSM. We then reinterpret the results of existing LHC direct searches for squarks when potentially altered squark decay modes are allowed. In Sect. 3, we present an analysis strategy targeting the specific channel where, after squark pair-production, one squark decays into a top quark and the second squark into a charm quark. Projections for sensitivity of the coming LHC runs to the model are presented and discussed in Sect. 4, where we emphasise the potential role of charm tagging. Our conclusions are given in Sect. 5.

\section{Model setup and existing LHC limits}

In this section, we describe the simplified model featuring top-charm mixing in the squark sector which we base our analysis on. We discuss general features of the search strategy for targeting such a model, and reinterpret recent squark searches to assess the current LHC coverage of the model parameter space.

\subsection{A simplified model for squark flavour violation}

In order to assess the LHC sensitivity to supersymmetric models featuring non-minimal flavour violation in the squark sector, we consider a simplified model embedding two active flavours of squarks, a right-handed top squark $\tilde{t}_{R}$ and a righthanded charm squark $\tilde{c}_{R}$. These two states mix into two physical eigenstates $\tilde{u}_{1}$ and $\tilde{u}_{2}$ whose flavour structure is dictated by the $\theta_{t c}$ mixing angle,

$$
\left(\begin{array}{c}
\tilde{u}_{1} \\
\tilde{u}_{2}
\end{array}\right)=\left(\begin{array}{rr}
\cos \theta_{t c} & \sin \theta_{t c} \\
-\sin \theta_{t c} & \cos \theta_{t c}
\end{array}\right)\left(\begin{array}{c}
\tilde{c}_{R} \\
\tilde{t}_{R}
\end{array}\right),
$$

where by convention $\tilde{u}_{1}$ is the lighter of the two mass eigenstates. Our simplified model additionally includes one neutralino $\tilde{\chi}_{1}^{0}$, that we take bino-like. Such an assumption does not have a significant impact on our phenomenological results. The setup of our interest is thus based on four parameters: the masses $m_{\tilde{u}_{1}}$ and $m_{\tilde{u}_{2}}$ of the two physical squarks together with the flavour mixing angle $\theta_{t c}$, and the neutralino mass $m_{\tilde{\chi}_{1}^{0}}$. For the sensitivity studies in the $\left(m_{\tilde{u}_{1}}, \theta_{t c}\right)$ plane, the neutralino mass will be fixed to $m_{\tilde{\chi}_{1}^{0}}=50 \mathrm{GeV}$. Although a more complicated flavour structure involving lefthanded squarks could be possible as well, this last setup implies the need to handle more complicated constraints from $B$-physics in order to build phenomenologically viable scenarios. Left-handed squarks are thus assumed heavier and decoupled, like any other superpartner.

Our simplified model therefore exhibits two competing squark decay modes (if kinematically allowed),

$\tilde{u}_{i} \rightarrow t \tilde{\chi}_{1}^{0}, \quad \tilde{u}_{i} \rightarrow c \tilde{\chi}_{1}^{0} \quad$ with $i=1,2$,

which yield three classes of LHC signatures originating from the production of a pair of $\tilde{u}_{i}$ squarks. Typical LHC search strategies have been designed on the basis of the MFV paradigm and thus only address the two signatures:

$p p \rightarrow t \bar{t}+E_{\mathrm{T}}^{\mathrm{miss}}$ and $p p \rightarrow c \bar{c}+E_{\mathrm{T}}^{\mathrm{miss}}$,

where $E_{\mathrm{T}}^{\text {miss }}$ is the imbalance in transverse momentum in the event generated by the undetected neutralinos. Squark flavour mixing opens up a third final state,

$p p \rightarrow t c+E_{\mathrm{T}}^{\mathrm{miss}}$,

where one squark decays into a top quark and the other one into a charm quark [29].

In Fig. 1, we illustrate the $\theta_{t c}$-dependence of the squark branching ratios associated with the decays of Eq. (2). We observe that regardless the squark and neutralino mass configuration, there always exists a $\theta_{t c}$ value for which both decay modes have $50 \%$ branching ratio, which means that half of the signal events would produce the final state of Eq. (4). Moreover, differences in the functional behaviour of the branching ratios for different mass hierarchies become only noticeable close to threshold, when the mass splitting 


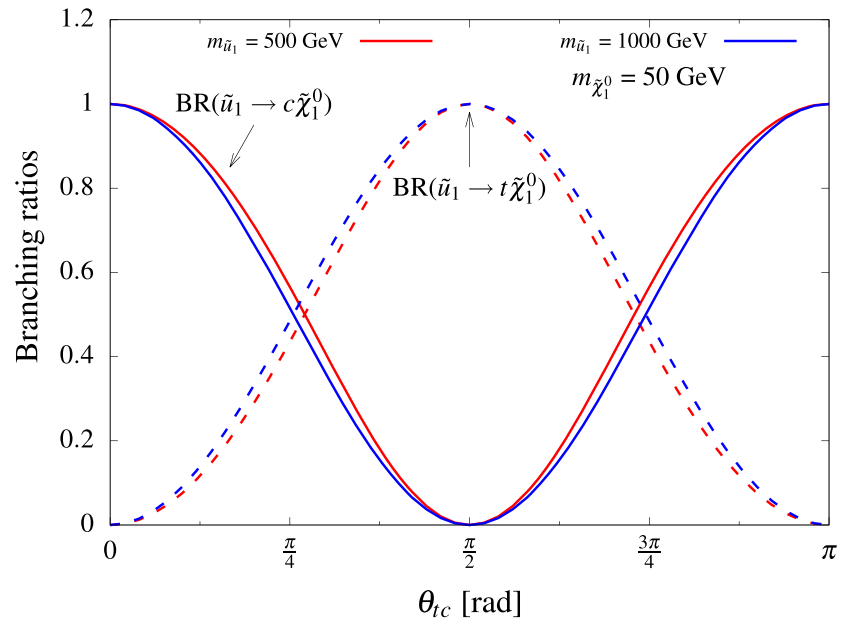

Fig. 1 Dependance of the branching ratios $\operatorname{BR}\left(\tilde{u}_{1} \rightarrow t \tilde{\chi}_{1}^{0}\right)$ (dashed) and $\operatorname{BR}\left(\tilde{u}_{1} \rightarrow c \tilde{\chi}_{1}^{0}\right)$ (solid) on the squark mixing angle $\theta_{t c}$ for various mass configurations. In the left panel, the squark mass is fixed to $500 \mathrm{GeV}$ (red) and $1000 \mathrm{GeV}$ (blue), with the neutralino mass being set

between the decaying squark and the neutralino is small. This configuration is not considered further in this paper, as the phase space available for the decay is limited and the best experimental sensitivity is achieved with monojet or monotop probes [38].

\subsection{Reinterpretation of current LHC Run 2 results}

The ATLAS and CMS collaborations have performed several direct searches for top squarks, mostly in a setup where they are pair-produced and decay into a pair of top-antitop quarks and missing energy as indicated by the first equation of Eq. (3). With no observation of any hint for new physics, the most stringent constraints arise from LHC Run 2 analyses of proton-proton collisions at a centre-of-mass energy of $13 \mathrm{TeV}$ [1-12]. All these searches lead to exclusion limits on the top squark mass of the order of $1 \mathrm{TeV}$. Bounds on first and second generation squarks are similar when one single light squark species is considered together with a decay into light jets and missing transverse energy, whereas they reach $1.5 \mathrm{TeV}$ for models featuring four mass-degenerate first and second generation squarks $[13,14]$. The most sensitive stop searches, yielding a similar expected sensitivity for low neutralino masses, are the ones addressing final states with either zero or one lepton. We therefore choose the recent ATLAS search for top squarks in final states with one lepton of Ref. [12] as a benchmark for getting conservative Run 2 constraints on our model.

Additionally, the ATLAS collaboration has carried out an analysis targeting top squarks decaying into charm and missing energy or charm squarks [15], based on the experimental tagging of jets produced from the fragmentation of

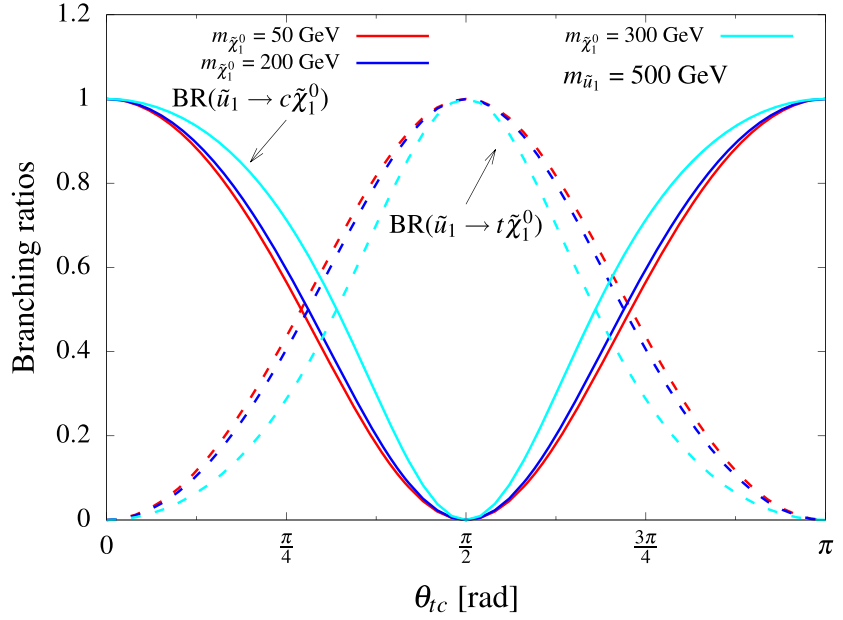

to $m_{\tilde{\chi}_{1}^{0}}=50 \mathrm{GeV}$. In the right panel, the neutralino mass varies and is fixed to $50 \mathrm{GeV}$ (red), $200 \mathrm{GeV}$ (blue) and $300 \mathrm{GeV}$ (cyan), for a squark mass of $m_{\tilde{u}_{1}}=500 \mathrm{GeV}$

charm quarks. As this signature is expected to play a significant role for getting handles on the considered squark inter-generational mixings, we use the analysis in Ref. [15] as a second LHC Run 2 benchmark to evaluate the existing constraints on our simplified model.

We perform a three-dimensional parameter space scan and vary independently the two squark masses $\left(m_{\tilde{u}_{1}}\right.$ and $\left.m_{\tilde{u}_{2}}\right)$, as well as the top-charm squark mixing angle $\theta_{t c}$. As mentioned above, the neutralino mass has been fixed to $50 \mathrm{GeV}$, so that our results are valid as long as the squark masses are much larger than the neutralino mass. For each considered point, we evaluate the sensitivity of the two searches of Refs. $[12,15]$ and present the results in Fig. 2. The excluded region lies between the exclusion contour and the left-side of the figure. Concerning the stop analysis [12], we rely on the acceptances and efficiencies that have been officially provided by the ATLAS collaboration for each of the 'discovery tN_med' (targeting moderate stop masses) and 'discovery tN_high' (targeting high stop masses) regions. We then estimate the two corresponding signal yields $\left(N_{\text {sig }}\right)$, considering next-toleading order (NLO) stop pair-production rates corrected by the resummation of the threshold logarithms at the next-toleading logarithmic (NLL) accuracy [39] and the appropriate branching ratios. These signal yields are then compared to the ATLAS model-independent upper limit $\left(N_{\text {non }}^{\text {obs limit }}\right)$ for each of the regions. If the ratio of these two yields exceeds one, the signal point is considered excluded. While providing acceptance and efficiency values only for the inclusive 'signal' regions, the ATLAS analysis employs a multi-bin fit in the most sensitive distribution for the final exclusion limit estimation. For this reason the recast exclusion contours presented in Fig. 2 represent a conservative estimate of 


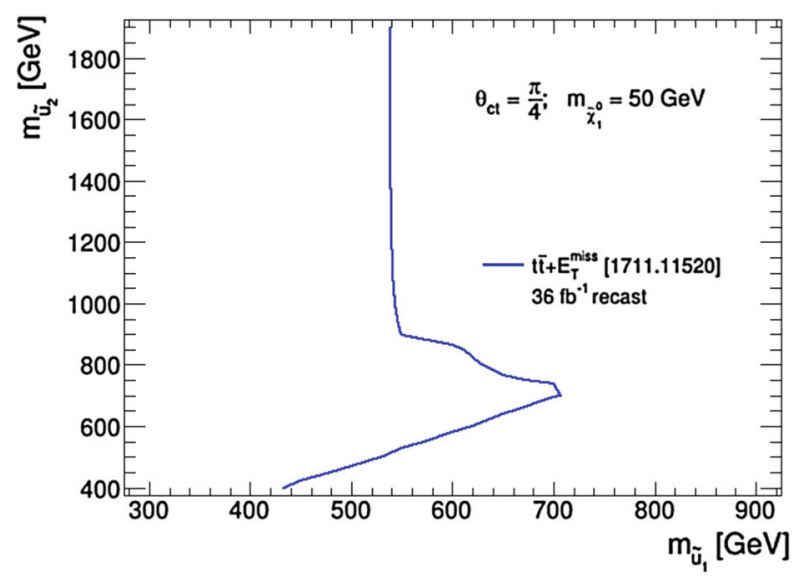

Fig. 2 Reinterpretation, in the context of our simplified model, of the ATLAS bounds on top squarks obtained with single-leptonic probes [12] and on Supersymmetry when charm tagging is used [15]. The results are presented in the $\left(m_{\tilde{u}_{1}}, m_{\tilde{u}_{2}}\right)$ plane (left) and $\left(m_{\tilde{u}_{1}}, \theta_{t c}\right)$ plane (right), and

the effective reach of the ATLAS search. We rely on the same procedure to extract the constraints from the charm-tagging analysis of Ref. [15].

In the left panel of Fig. 2, we consider a class of benchmark scenarios where the two squark eigenstates are maximal admixtures of the top and charm flavours $\left(\theta_{c t}=\frac{\pi}{4}\right)$ and we vary the two masses independently (with $m_{\tilde{u}_{1}}<m_{\tilde{u}_{2}}$ ). The total new physics production rate is here solely driven by the lightest of the two states, except for the region where the mass splitting of the two squarks is small. For sufficiently high splittings, the exclusion is thus independent of $m_{\tilde{u}_{2}}$, and squarks are found to be constrained to be heavier than about $550 \mathrm{GeV}$. Compared with the more standard MFV case where the two eigenstates are also flavour eigenstates (and where the bounds are of about $1 \mathrm{TeV}$ ), the limits are hence weakened by almost $500 \mathrm{GeV}$. The large value of the top-charm mixing angle indeed implies that the two signal regions of the stop analysis of Ref. [12], specifically targeting final states with the decay products of two top quarks, are less populated by virtue of the large decay fraction into charm jets $\operatorname{BR}\left(\tilde{u}_{1} \rightarrow c \tilde{\chi}_{1}^{0}\right)$. In the parameter space region defined by

$m_{\tilde{u}_{1}}, m_{\tilde{u}_{2}} \lesssim 750 \mathrm{GeV}$,

the situation is somewhat different as the two squark mass eigenstates contribute to a potentially observable new physics signal. This partly compensates the loss due to the smaller branching ratio into tops, so that the obtained limits are stronger than when the second eigenstates is heavier. The charm-tagging analysis of Ref. [15] always implies weaker bounds for this specific classes of scenarios (the number of events populating the signal regions being very small), and the corresponding results are thus omitted.

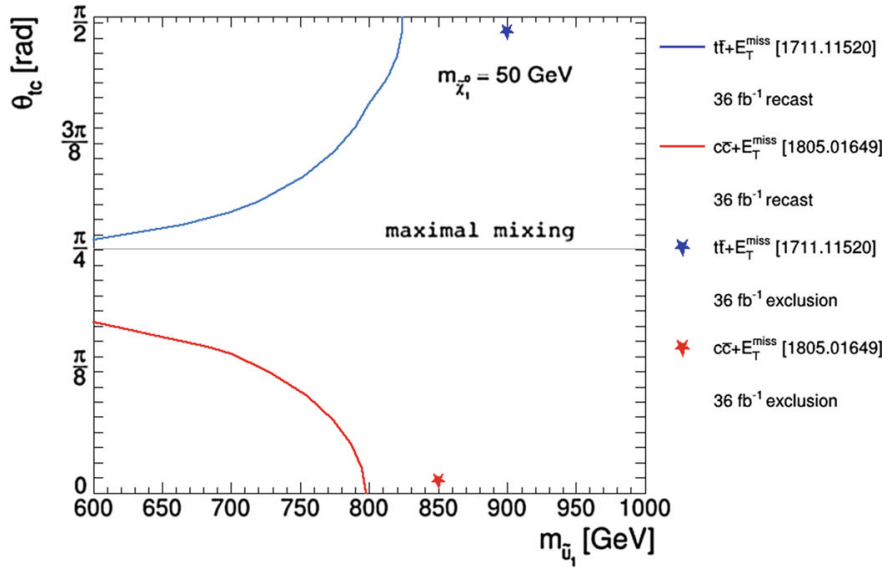

the stars correspond to the official ATLAS results in the non-flavourmixing case. The excluded region lies between the exclusion contour and the left-side of the figure

In the right panel of Fig. 2, we reinterpret the ATLAS limits in the $\left(m_{\tilde{u}_{1}}, \theta_{t c}\right)$ plane, i.e. we decouple the second eigenstate. Our results exhibit the complementary effect of the top-charm squark mixing angle on the bounds. For $\theta_{t c}=0$, the lightest squark is purely of charm flavour, so that the ATLAS stop search is insensitive to the signal and the limits $\left(m_{\tilde{u}_{1}} \gtrsim 800 \mathrm{GeV}\right)$ solely arise from the ATLAS charm-tagging analysis. With the mixing angle increasing, the $c \bar{c}+E_{\mathrm{T}}^{\text {miss }}$ production rate decreases so that the bounds are progressively weakened. On the other hand, the increase in $\theta_{t c}$ implies that while the signal regions of the charmtagging analysis are more and more depleted due to the lower and lower $\operatorname{BR}\left(\tilde{u}_{1} \rightarrow c \tilde{\chi}_{1}^{0}\right)$ branching ratio, the signal regions of the stop analysis are more and more populated due to the increasing $\operatorname{BR}\left(\tilde{u}_{1} \rightarrow t \tilde{\chi}_{1}^{0}\right)$ branching ratio. In the limit for which the lightest squark is purely of top flavour, its mass is constrained to be at least $825 \mathrm{GeV}$. In the maximal-mixing condition, the mass constraints for both analyses are below $600 \mathrm{GeV}$, which is the minimum mass value for which experimental acceptances are available for both the considered benchmark analyses.

We superimpose to the results of our recasting the official limits observed by ATLAS, represented by stars on the right panel of Fig. 2 for the cases where the lightest squark is of a definite flavour. The usage of multi-bin signal regions increases the limits by about $50-100 \mathrm{GeV}$.

\section{Collider projections for the reach of the $t c$ channel}

We have shown in the previous section that the current experimental searches focusing on pair production of squarks that carry a well-defined flavour have a significantly reduced sen- 
sitivity to models with sizeable values of flavour mixing. The issue may be addressed by developing a dedicated analysis targeting the $t c+E_{\mathrm{T}}^{\text {miss }}$ channel, which has its maximum rate in the case of maximum mixing. We describe a possible implementation of such an analysis in this section. In particular, we will focus on the case in which the top quark decays semileptonically, resulting in a final state with an isolated lepton, a $b$-jet, a $c$-jet and missing transverse energy.

Our study assumes proton-proton collisions at a centre-ofmass energy of $14 \mathrm{TeV}$, and integrated luminosities of 300 and $3000 \mathrm{fb}^{-1}$, corresponding to the expected configurations for the coming LHC runs.

\subsection{Monte Carlo simulation}

In order to simulate our signal, we have implemented the model of Sect. 2.1 into FEYNRULES 2.0 [40] to get a UFO model [41] to be used within the MADGRAPH5_aMC@NLO framework [42]. We have generated leading-order (LO) hardscattering matrix elements for squark pair-production and decay, that we have convoluted with the leading-order set of NNPDF 3.0 parton distribution functions [43]. Parton showering and hadronisation have been handled with PYTHIA 8.2 [44], and each event has been reweighted so that the corresponding total rate matches the production cross-section estimated at the NLO + NLL accuracy [39]. We generate a grid in the parameter space of the model, the lightest squark mass being varied in the $[600 \mathrm{GeV}, 1.5 \mathrm{TeV}]$ window by steps of $100 \mathrm{GeV}$, and the neutralino mass in the $[50 \mathrm{GeV}$, $900 \mathrm{GeV}$ ] window in steps of $50 \mathrm{GeV}$ for $m_{\tilde{\chi}_{1}^{0}}<400 \mathrm{GeV}$ and of $100 \mathrm{GeV}$ above. The squark mixing angle is fixed to $\pi / 4$.

As stated above, we focus on the signal topology with one isolated lepton (electron or muon), jets and missing transverse energy. The SM processes which can mimic this topology involve one or two leptons originating either from the decay of a $W$ or a $Z$ boson, or from leptonically-decaying tau leptons. We consequently generate events for $\mathrm{SM} t \bar{t}, W t$, $t$-channel single top, $t \bar{t} W, t \bar{t} Z, t W Z, t Z, W+$ jets, $Z+$ jets, $W W, W Z$ and $Z Z$ production. For $t \bar{t}$, single top and diboson processes, events are simulated at the NLO in QCD within the POWHEG Box framework [45]. Samples for the remaining processes are then generated at LO, using MADGRAPH5_aMC@NLO. We consider matrix elements featuring a variable number of additional jets that we merge according to the CKKW prescription as implemented in PYTHIA 8 [46]. For $W+$ jets and $Z+$ jets, we merge samples describing final-states containing up to four additional partons, whereas for $t \bar{t} W$ and $t \bar{t} Z$ production, the matrix elements are allowed to include up to two extra partons. All those events are reweighted so that the total rates match the next-to-next-toleading order (NNLO) cross-sections if available, or the NLO ones otherwise.
Jets are reconstructed according to the anti- $k_{T}$ jet algorithm [47] with a jet radius parameter set to $R=0.4$, as implemented in FASTJET [48]. Moreover, jets are labelled as $b$-jets if the angular distance $\Delta R \equiv\left(\Delta \phi^{2}+\Delta \eta^{2}\right)^{1 / 2}$ between the jet and the nearest $B$-hadron satisfies $\Delta R<0.5$. Similarly, we define $c$-jets as jets that fail $b$-tagging and for which there exists a charmed hadron lying at an angular distance smaller than 0.5 from the jet. Any jet that is not identified as a $b$-jet or as a $c$-jet is labelled as a light jet. The missing transverse momentum $\mathbf{p}_{\mathrm{T}}^{\text {miss }}$, with magnitude $E_{\mathrm{T}}^{\text {miss }}$, is estimated by the vector sum of the transverse momenta of all invisible particles.

Detector effects are simulated by smearing the momenta of all reconstructed objects and by applying reconstruction efficiency factors in a way that reproduces the performance of the ATLAS detector [49,50], as described in Ref. [51]. In particular, we include $b$-tagging and $c$-tagging efficiency and rejection factors based on the performance reported in Refs. $[52,53]$, and we adopt working points corresponding to an average $b$-tagging efficiency of $\epsilon_{b}(b)=77 \%$ for charm and light jet rejection factors of $1 / \epsilon_{b}(c)=4.5$ and $1 / \epsilon_{b}(l)=140$ respectively, and an average $c$-tagging efficiency of $\epsilon_{c}(c)=$ $30 \%$ for rejection factors of $1 / \epsilon_{c}(b)=18$ and $1 / \epsilon_{c}(l)=5$ for $b$-jets and light jets respectively. Such a choice is aimed at optimising background rejection when it is dominated by final states featuring two $b$-jets. As there is currently no public information on the correlations between the $b$-tagging and $c$-tagging algorithms used by the collaborations, we do not allow a jet to be $b$-tagged and $c$-tagged simultaneously. We instead first select jet candidates based on their kinematics before applying either $b$-tagging or $c$-tagging.

We have compared our approach with an independent simulation based on the publicly available detector simulation software DELPHES 3 [54], and have found good agreement between the two methods.

\subsection{Event selection}

The topology of interest includes one isolated lepton (electron or muon) arising from the top decay, jets including one $b$-jet (also issued from the top decay) and one $c$-jet, as well as missing transverse energy carried by the two neutralinos. Consequently, we preselect events by requiring the presence of exactly one isolated electron or muon with a transverse momentum $p_{\mathrm{T}}>25 \mathrm{GeV}$ and a pseudorapidity $|\eta|<2.5$, and of at least one $b$-tagged jet with $p_{\mathrm{T}}>50 \mathrm{GeV}$ and $|\eta|<2.5$. We moreover ask the invariant mass of at least one of the possible systems made of a $b$-jet and the lepton to fulfil $m_{b \ell}<160 \mathrm{GeV}$, since in the signal case the lepton and the $b$-jet originate from a top decay so that $m_{b \ell}$ is bounded to be smaller than around $153 \mathrm{GeV}$.

The dominant backgrounds at this point are comprised of $t \bar{t}$ events with either one or both top quarks decaying lep- 
tonically, single top events and $t \bar{t} Z$ events with an invisible $Z$-boson decay. As all backgrounds where the missing energy originates from a leptonic $W$-boson decay $(W \rightarrow \ell v)$ feature

$m_{\mathrm{T}}^{l e p} \equiv \sqrt{2\left|\mathbf{p}_{\mathrm{T}}^{\ell}\right| E_{\mathrm{T}}^{\text {miss }}\left(1-\cos \Delta \phi\left(\mathbf{p}_{\mathrm{T}}^{\ell}, \mathbf{p}_{\mathrm{T}}^{\text {miss }}\right)\right)}<m_{W}$,

we require $m_{\mathrm{T}}^{\text {lep }}>160 \mathrm{GeV}$ to increase the signal over background ratio. In the definition of $m_{\mathrm{T}}^{l e p}, \mathbf{p}_{\mathrm{T}}^{\ell}$ is the lepton (vector) transverse momentum and $\Delta \phi\left(\mathbf{p}_{\mathrm{T}}^{\ell}, \mathbf{p}_{\mathrm{T}}^{\text {miss }}\right)$ the angle between $\mathbf{p}_{\mathrm{T}}^{\ell}$ and $\mathbf{p}_{\mathrm{T}}^{\text {miss }}$. Moreover, most of these backgrounds exhibit two $b$-jets in the final state, whereas the signal features in contrast one $b$-jet and one $c$-jet. Two strategies can therefore be envisaged to separate the signal from the backgrounds. Either one could veto the presence of any additional $b$-tagged jet besides the one required at the preselection level (Case-A), or one could enforce, in addition, the presence of an extra $c$-tagged jet (Case-B). From naive calculations based on the efficiencies of the different tagging algorithms, the signal over background ratio is improved by a factor of about 1.5 more for the Case-B strategy, but at the price of an overall reduction in statistics by a factor of approximately 3 . Both approaches are thus pursued in the following. For the Case-A strategy, we veto the presence of any extra $b$-jet and impose that there is an extra light jet with $p_{\mathrm{T}}>100 \mathrm{GeV}$ failing $b$ tagging. In contrast, for the Case-B strategy, we require that only one $b$-tagged jet satisfies $m_{b \ell}<160 \mathrm{GeV}$, we additionally impose that the leading jet fulfilling $m_{j \ell}>160 \mathrm{GeV}$ is $c$-tagged and has a $p_{\mathrm{T}}>100 \mathrm{GeV}$, and we ask all remaining jets with $m_{j \ell}>160 \mathrm{GeV}$ to fail $b$-tagging.

In order to further reduce the dileptonic $t \bar{t}$ background where one of the leptons escapes identification, we make use of the now standard asymmetric $m_{\mathrm{T} 2}$ variable (denoted $\left.a m_{\mathrm{T} 2}\right)[55,56]$ that consists in a variant of the $m_{\mathrm{T} 2}$ observable. The $a m_{\mathrm{T} 2}$ variable is built from two legs (corresponding to the two decay chains) containing both a visible part and an invisible part, and it requires two test masses corresponding to the invisible mass attached with each leg. The visible part of the first leg is built using the sum of the momenta of the $b$-tagged jet and of the lepton, with a test mass that is set to zero. The visible part of the second leg is built from the remaining jet with the highest $b$-tagging weight and $m_{W}$ is used as a test mass. The targeted background distribution featuring an end-point at approximately $160 \mathrm{GeV}$, we impose an $a m_{\mathrm{T} 2}>200 \mathrm{GeV}$ cut. In addition, the background can be further reduced by constructing another transverse $m_{T 2}$ variable. The signal topology features one squark leg where there is a hard $c$-jet, such that the distribution in the transverse mass built from the transverse momentum of the $c$-jet and the one of the neutralino exhibits an end-point at $\left(m_{\tilde{u}_{1}}^{2}-m_{\tilde{\chi}_{1}^{0}}^{2}\right)^{1 / 2}$. This feature can be exploited by constructing an appropriate $m_{\mathrm{T} 2} b l j$ variable. The visible part of the first leg is built from the sum of the momenta of the $b$-tagged jet and of the lepton, together with a vanishing test mass. The visible part of the second leg uses the hardest non- $b$-tagged jet or the $c$-tagged jet for the Case-A and Case-B strategies respectively, and again a vanishing test mass. We impose a selection on $m_{\mathrm{T} 2} b l j$ depending on the squark-neutralino mass splitting in order to optimise the sensitivity to the signal. This optimisation is performed by varying the cut threshold from 300 to $600 \mathrm{GeV}$ in steps of $50 \mathrm{GeV}$.

Finally, it is found that after all cuts, the missing transverse momentum is aligned with one of the jets of the event for the backgrounds, whereas there is no preferential direction for the signal. We therefore apply a cut on the minimum azimuthal angle separation between any jet and the missing transverse momentum, $\left|\Delta \phi_{\min }\right|>0.6$.

As an illustration, we present, in Fig. 3, the distribution in the $m_{\mathrm{T} 2_{b l j}}$ (left) and $\left|\Delta \phi_{\min }\right|$ variable (right) for the different SM backgrounds and two representative signal benchmark points. All selection cuts from the Case-A analysis strategy are imposed, but the one on the represented variable. On the left panel, we can observe that a selection of $m_{\mathrm{T} 2} b l j$ $>400 \mathrm{GeV}$ is sufficient to separate the signal from the background for the lighter of the chosen benchmark models. On the right panel, we show instead the $\left|\Delta \phi_{\min }\right|$ distribution after including a cut of $400 \mathrm{GeV}$ on $m_{\mathrm{T} 2} b_{l j}$. We can again observe that a significant improvement of the signal to background ratio can be achieved by imposing a $\left|\Delta \phi_{\min }\right|>0.6$ cut.

\section{Results}

On the basis of the analysis strategy outlined in the previous section, we estimate the LHC sensitivity to supersymmetric scenarios featuring mixed stop-scharm states with $300 \mathrm{fb}^{-1}$ and $3000 \mathrm{fb}^{-1}$ of integrated luminosity. For the latter configuration, we assume no modification in the detector performances for the high-luminosity LHC. The sensitivity is extracted by means of a test statistics based on a profiled likelihood ratio, and we make use of the CLs method [57] to obtain 95\% confidence level (CL) exclusion limits. The statistical analysis is performed with the ROOSTAT toolkit [58] and we assume systematic uncertainties of $20 \%$ and $5 \%$ on the SM backgrounds and on the signal respectively. The results are presented in terms of the upper limits, at the $95 \% \mathrm{CL}$, on the ratio of the signal yields to the corresponding benchmark predictions, denoted as $\sigma^{\text {excl }} / \sigma^{\text {SUSY }}$.

We show in the left panel of Fig. 4 the analysis reach in the $\left(m_{\tilde{u}_{1}}, m_{\tilde{\chi}_{1}^{0}}\right)$ plane both for the Case-A (red) and Case$\mathrm{B}$ (blue) analysis strategies and for $300 \mathrm{fb}^{-1}$ (solid) and $3000 \mathrm{fb}^{-1}$ (dashed) of integrated luminosity. The region that lies between the exclusion contour and the bottom-left side of the figure will be excluded at the future runs of LHC. The expected $95 \%$ upper limit on $m_{\tilde{u}_{1}}$ for $m_{\tilde{\chi}_{1}^{0}}=50 \mathrm{GeV}$ is 


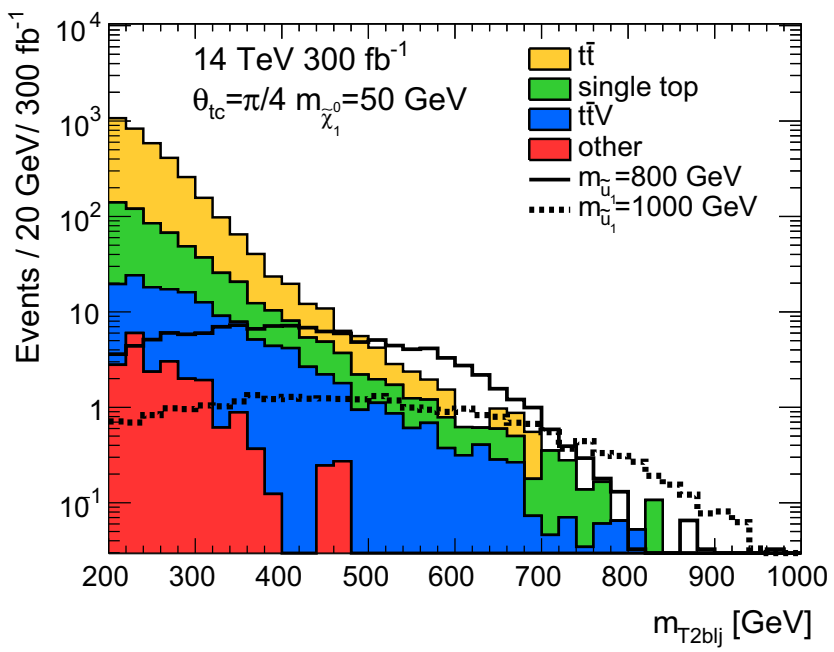

Fig. 3 Distributions in the $m_{\mathrm{T} 2 b l j}$ (left) and $\left|\Delta \phi_{\min }\right|$ (right) variables after imposing all cuts of the Case-A analysis strategy, excepted the one corresponding to the represented variable. The different background

LHC $14 \mathrm{TeV}$

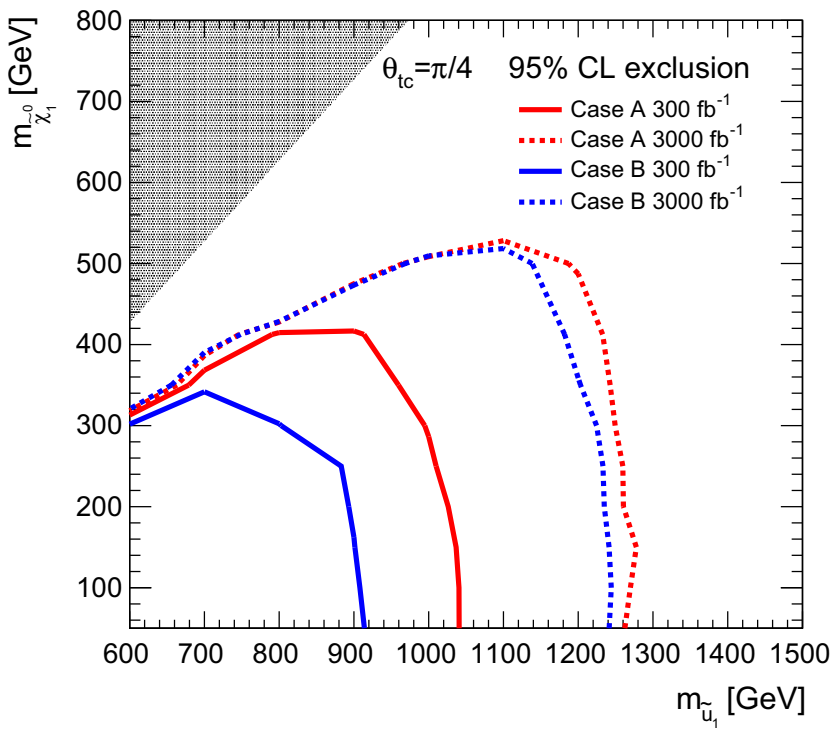

Fig. 4 Left: sensitivity of the LHC to our mixed stop-scharm scenarios given as $95 \% \mathrm{CL}$ exclusion contours in the $\left(m_{\tilde{u}_{1}}, m_{\tilde{x}_{1}^{0}}\right)$ plane for the Case-A (red) and Case-B (blue) analysis strategies and for $300 \mathrm{fb}^{-1}$ (solid) $3000 \mathrm{fb}^{-1}$ (dashed). The projected excluded region lies between

$1050 \mathrm{GeV}$ for Case-A, and $920 \mathrm{GeV}$ for Case-B for an integrated luminosity of $300 \mathrm{fb}^{-1}$. The large difference is due to the fact that the analysis reach is in this case dominated by statistics, which is lower for the analysis based on $c$-tagging, due to the $30 \%$ efficiency of the chosen $c$-tagging working point. For an integrated luminosity of $3 \mathrm{ab}^{-1}$ the difference is reduced, with a reach of $1280 \mathrm{GeV}$ for Case-A and $1240 \mathrm{GeV}$ for Case-B.

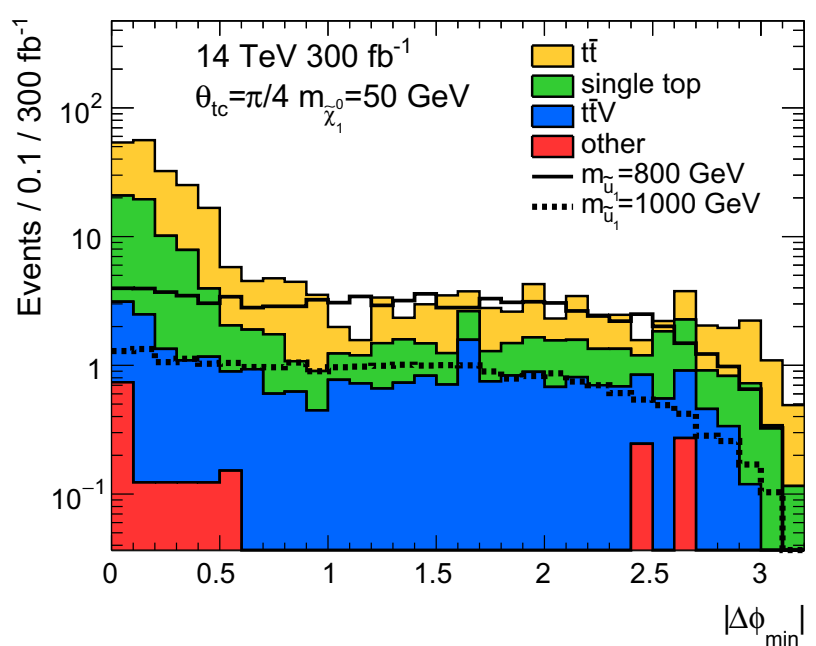

contributions and two representative signal scenarios are shown for an integrated luminosity of $300 \mathrm{fb}^{-1}$. For the $\left|\Delta \phi_{\min }\right|$ distribution, the $m_{\mathrm{T} 2 b l j}$ variable is required to be larger than $400 \mathrm{GeV}$

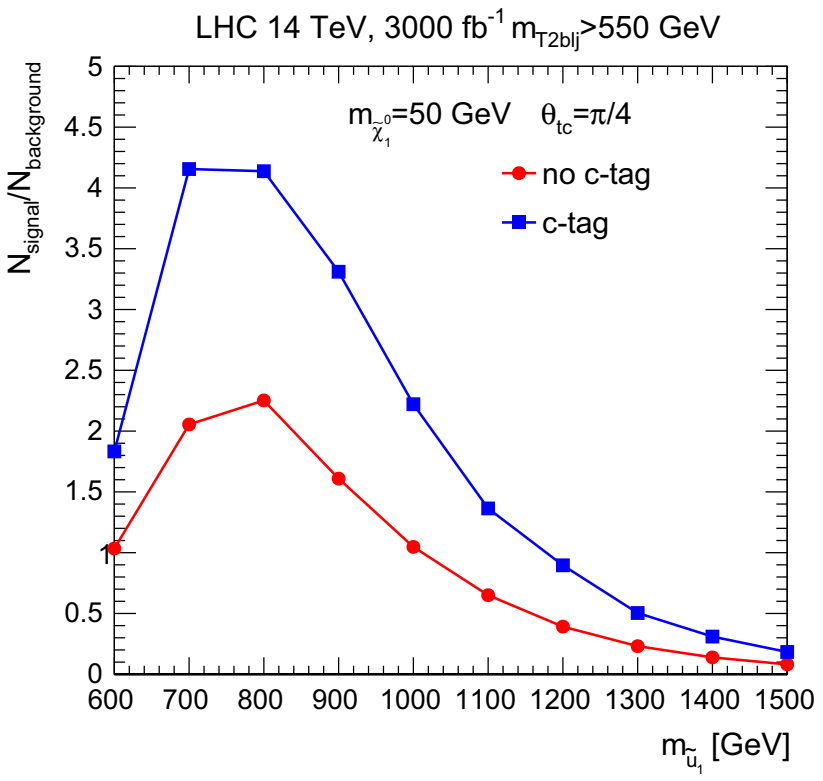

the exclusion contour and the bottom-left side of the figure. Right: signal over background ratio as a function of $m_{\tilde{u}_{1}}$ for the Case-A (red, rounds) and Case-B (blue, squares) analysis strategies when one imposes that $m_{\mathrm{T} 2_{b l j}}>550 \mathrm{GeV}$

In order to better understand the relative performance of the two analysis strategies, we present in the right panel of Fig. 4 the dependence of the signal over background ratio $(S / B)$ on the squark mass $m_{\tilde{u}_{1}}$ for $m_{\tilde{\chi}_{1}^{0}}=50 \mathrm{GeV}, \theta_{t c}=\pi / 4$ and when a $m_{\mathrm{T} 2_{b l j}}>550 \mathrm{GeV}$ cut is applied. As expected, the $S / B$ ratio is higher when $c$-tagging is incorporated. Comparisons of results stemming from analyses with and without $c$-tagging, or relying on different $c$-tagging working points 


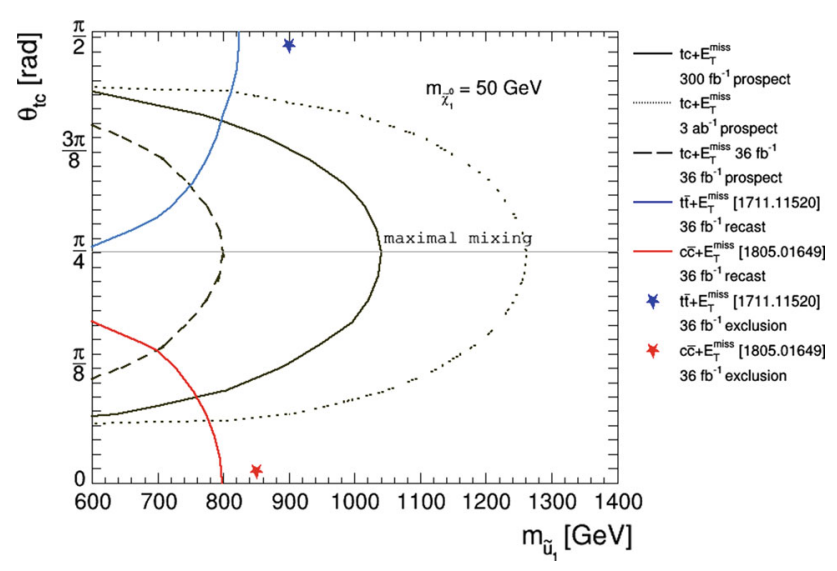

Fig. 5 Present and expected exclusion limits in the $\left(\theta_{t c}, m_{\tilde{u}_{1}}\right)$ plane. The area between the recasted exclusion contour and the top-left (bottom-left) side of the figure is excluded by the $t \bar{t}+E_{\mathrm{T}}^{\text {miss }}(c \bar{c}+$ $E_{\mathrm{T}}^{\mathrm{miss}}$ ) analysis. The region that lies between the exclusion contour (solid, dashed and dotted black lines) and the left side of the figure will be excluded at the future runs of LHC using $t c+E_{\mathrm{T}}^{\text {miss }}$ analysis. See the text for details

could be used to get information on the flavour content of the observed squark, which is the main information one would like to extract in case of a discovery. In the Case-B analysis, we have chosen a $c$-tagging working point which optimises $S / B$, but with a similar efficiency for $c$-jets and light jets, and thus not ideal for discriminating the flavour of the signal. A different $c$-tagging algorithm working point featuring a very high rejection for light jets, as e.g. in Ref. [15] with $\epsilon_{c}(c)=18 \%, 1 / \epsilon_{c}(b)=20$ and $1 / \epsilon_{c}(l)=200$ would yield a lower overall sensitivity, but might be used to discriminate between different flavour mixing hypotheses for the signal.

In Fig. 5, we show the 95\% CL exclusion limits in the $\left(m_{\tilde{u}_{1}}, \theta_{t c}\right)$ plane for a fixed neutralino mass of $50 \mathrm{GeV}$. Recasts of the $13 \mathrm{TeV}$ exclusion limits obtained by the ATLAS experiment with $36 \mathrm{fb}^{-1}$ are in addition overlaid (see Sec. 2.2), the blue curve corresponding to the ATLAS search for stop in the singly-leptonic mode [12] and the red one to the ATLAS search for squarks based on charm-tagging [15]. The region between the recasted exclusion contour and the top-left (bottom-left) side of the figure is excluded by the $t \bar{t}+E_{\mathrm{T}}^{\mathrm{miss}}\left(c \bar{c}+E_{\mathrm{T}}^{\mathrm{miss}}\right)$ analysis. The exclusion limits expected from the $t c+E_{\mathrm{T}}^{\text {miss }}$ Case-A analysis strategy are shown as solid and dotted black lines for integrated luminosities of $300 \mathrm{fb}^{-1}$ and $3000 \mathrm{fb}^{-1}$ respectively. Moreover, we also include the expectation of such an analysis at $14 \mathrm{TeV}$, with $36 \mathrm{fb}^{-1}$ of luminosity. For $t c+E_{\mathrm{T}}^{\text {miss }}$ analysis, the projected excluded region lies between the exclusion contour (solid, dashed and dotted black lines) and the left side of the figure. This figure clearly illustrates the strength of the $t c$ $+E_{\mathrm{T}}^{\mathrm{miss}}$ analysis we are proposing, covering a region of the parameter space not accessible with current searches relying on the MFV paradigm.

\section{Summary}

We have studied a supersymmetric scenario departing from the traditional MFV paradigm. Flavour mixing including squarks of the first generation and left-handed partners being strongly constrained by data, we focus on specific scenarios where right-handed stop and scharm mix. In this context, squark pair production and decay can yield three distinctive signatures, namely top pairs plus missing energy, charm-jet pairs with missing energy, and $t c$ plus missing energy.

By recasting existing LHC searches for top and charm squarks, we have shown that a significant fraction of the parameter space is evading present LHC constraints and that mixed squark states with masses around $600 \mathrm{GeV}$ remain a phenomenologically viable option. This is in particular true when the top-charm mixing is maximal, as both $t \bar{t}+E_{\mathrm{T}}^{\text {miss }}$ and $c \bar{c}+E_{\mathrm{T}}^{\text {miss }}$ signals are weakened. This apparent loophole in the experimental searches could be filled by the design of a dedicated $t c+E_{\mathrm{T}}^{\text {miss }}$ search for squarks.

To this purpose, we have developed two search strategies targeting the production of squarks containing two flavour components, both involving leptons, $b$-tagged jet and $E_{\mathrm{T}}^{\text {miss }}$. The core difference between them consists in using charmtagging in one of them, making hence use of the presence of a charm jet in the final state as a handle on new physics. With charm-tagging efficiencies presently achieved by experiments being lower than for $b$-tagging, the requirement of a charm-tagged jet implies a loss of signal statistics and hence of mass reach for an integrated luminosity of $300 \mathrm{fb}^{-1}$. The statistical limitation on the signal are however less important for the high-luminosity phase of the LHC, where mixed squarks as heavy as $1.3 \mathrm{TeV}$ could be targeted for both analysis strategies. The advantage of pursuing several analyses in parallel is that the comparison of the results from analyses relying on different requirement and/or working points for the flavour tagging of jets opens the door to probing the squark flavour structure and hence allows the potential observation of departures from the MFV paradigm.

As charm tagging is being actively developed by both the ATLAS and CMS collaborations, we urge them to add to their search program a dedicated $t c+E_{\mathrm{T}}^{\text {miss }}$ analysis that could provide sensitivity to new physics scenarios where the top partners are not flavour eigenstates.

Acknowledgements The authors would like to thank the organizers of 'Physics at TeV Colliders' workshop (Les Houches, June 2017) where this work was initiated. We would also like to thank Michihisa Takeuchi for many useful discussions. This work has been partially supported by French state funds managed by the Agence Nationale de la Recherche (ANR) in the context of the Investissements d'avenir Labex ENIGMASS (ANR-11-LABX-0012) and Labex ILP (ANR-11-IDEX0004-02, ANR-10-LABX-63), and by the Grant-in-Aid for Scientific Research on Scientific Research B (No. 16H03991) and Innovative Areas (16H06492). 
Open Access This article is distributed under the terms of the Creative Commons Attribution 4.0 International License (http://creativecomm ons.org/licenses/by/4.0/), which permits unrestricted use, distribution, and reproduction in any medium, provided you give appropriate credit to the original author(s) and the source, provide a link to the Creative Commons license, and indicate if changes were made.

Funded by SCOAP ${ }^{3}$.

\section{References}

1. A.M. Sirunyan et al., Eur. Phys. J. C 77(10), 710 (2017). https:// doi.org/10.1140/epjc/s10052-017-5267-x

2. M. Aaboud, JHEP 09, 084 (2017). https://doi.org/10.1007/ JHEP09(2017)084

3. A.M. Sirunyan, JHEP 10, 019 (2017). https://doi.org/10.1007/ JHEP10(2017)019

4. A.M. Sirunyan, JHEP 10, 005 (2017). https://doi.org/10.1007/ JHEP10(2017)005

5. A.M. Sirunyan et al., Phys. Lett. B 778, 263 (2018). https://doi. org/10.1016/j.physletb.2018.01.012

6. M. Aaboud et al., Eur. Phys. J. C 77(12), 898 (2017). https://doi. org/10.1140/epjc/s10052-017-5445-x

7. M. Aaboud, JHEP 11, 195 (2017). https://doi.org/10.1007/ JHEP11(2017)195

8. M. Aaboud, JHEP 12, 085 (2017). https://doi.org/10.1007/ JHEP12(2017)085

9. A.M. Sirunyan et al., Phys. Rev. D 97(1), 012007 (2018). https:// doi.org/10.1103/PhysRevD.97.012007

10. A.M. Sirunyan et al., Phys. Rev. D 97(3), 032009 (2018). https:// doi.org/10.1103/PhysRevD.97.032009

11. M. Aaboud, JHEP 01, 126 (2018). https://doi.org/10.1007/ JHEP01(2018)126

12. M. Aaboud, JHEP 06, 108 (2018). https://doi.org/10.1007/ JHEP06(2018)108

13. M. Aaboud et al., Phys. Rev. D 97(11), 112001 (2018). https://doi. org/10.1103/PhysRevD.97.112001

14. A.M. Sirunyan et al., Phys. Rev. D 96(3), 032003 (2017). https:// doi.org/10.1103/PhysRevD.96.032003

15. M. Aaboud, JHEP 09, 050 (2018). https://doi.org/10.1007/ JHEP09(2018)050

16. S.P. Martin, Phys. Rev. D 75, 115005 (2007). https://doi.org/10. 1103/PhysRevD.75.115005

17. J. Fan, M. Reece, J.T. Ruderman, JHEP 11, 012 (2011). https://doi. org/10.1007/JHEP11(2011)012

18. H. Murayama, Y. Nomura, S. Shirai, K. Tobioka, Phys. Rev. D 86, 115014 (2012). https://doi.org/10.1103/PhysRevD.86.115014

19. M. Blanke, G.F. Giudice, P. Paradisi, G. Perez, J. Zupan, JHEP 06, 022 (2013). https://doi.org/10.1007/JHEP06(2013)022

20. G. Brooijmans, et al., in 10th Les Houches Workshop on Physics at TeV Colliders (PhysTeV 2017) Les Houches, France, June 5-23, 2017 (2018). http://lss.fnal.gov/archive/2017/conf/ fermilab-conf-17-664-ppd.pdf

21. M. Ciuchini, A. Masiero, P. Paradisi, L. Silvestrini, S.K. Vempati, O. Vives, Nucl. Phys. B 783, 112 (2007). https://doi.org/10.1016/ j.nuclphysb.2007.05.032

22. K. De Causmaecker, B. Fuks, B. Herrmann, F. Mahmoudi, B. O'Leary, W. Porod, S. Sekmen, N. Strobbe, JHEP 11, 125 (2015). https://doi.org/10.1007/JHEP11(2015)125

23. M. Dimou, S.F. King, C. Luhn, JHEP 02, 118 (2016). https://doi. org/10.1007/JHEP02(2016)118

24. M. Dimou, S.F. King, C. Luhn, Phys. Rev. D 93(7), 075026 (2016). https://doi.org/10.1103/PhysRevD.93.075026

25. G. Bozzi, B. Fuks, B. Herrmann, M. Klasen, Nucl. Phys. B 787, 1 (2007). https://doi.org/10.1016/j.nuclphysb.2007.05.031
26. B. Fuks, B. Herrmann, M. Klasen, Nucl. Phys. B 810, 266 (2009). https://doi.org/10.1016/j.nuclphysb.2008.11.020

27. T. Hurth, W. Porod, JHEP 08, 087 (2009). https://doi.org/10.1088/ 1126-6708/2009/08/087

28. M. Bruhnke, B. Herrmann, W. Porod, JHEP 09, 006 (2010). https:// doi.org/10.1007/JHEP09(2010)006

29. A. Bartl, H. Eberl, B. Herrmann, K. Hidaka, W. Majerotto, W. Porod, Phys. Lett. B 698, 380 (2011). https://doi.org/10.1016/j. physletb.2011.04.051. https://doi.org/10.1016/j.physletb.2011.01. 020.[Erratum: Phys. Lett. B 700, 390 (2011)]

30. A. Bartl, H. Eberl, E. Ginina, B. Herrmann, K. Hidaka, W. Majerotto, W. Porod, Phys. Rev. D 84, 115026 (2011). https://doi. org/10.1103/PhysRevD.84.115026

31. A. Bartl, H. Eberl, E. Ginina, B. Herrmann, K. Hidaka, W. Majerotto, W. Porod, Int. J. Mod. Phys. A 29(07), 1450035 (2014). https://doi.org/10.1142/S0217751X14500353

32. M. Backović, A. Mariotti, M. Spannowsky, JHEP 06, 122 (2015). https://doi.org/10.1007/JHEP06(2015)122

33. M. Blanke, B. Fuks, I. Galon, G. Perez, JHEP 04, 044 (2016). https://doi.org/10.1007/JHEP04(2016)044

34. A. Crivellin, U. Haisch, L.C. Tunstall, JHEP 09, 080 (2016). https:// doi.org/10.1007/JHEP09(2016)080

35. M. Blanke, S. Kast, JHEP 05, 162 (2017). https://doi.org/10.1007/ JHEP05(2017)162

36. M. Blanke, S. Das, S. Kast, JHEP 02, 105 (2018). https://doi.org/ 10.1007/JHEP02(2018)105

37. J.A. Evans, D. Shih, A. Thalapillil, JHEP 07, 040 (2015). https:// doi.org/10.1007/JHEP07(2015)040

38. B. Fuks, P. Richardson, A. Wilcock, Eur. Phys. J. C 75(7), 308 (2015). https://doi.org/10.1140/epjc/s10052-015-3530-6

39. C. Borschensky, M. Krämer, A. Kulesza, M. Mangano, S. Padhi, T. Plehn, X. Portell, Eur. Phys. J. C 74(12), 3174 (2014). https:// doi.org/10.1140/epjc/s10052-014-3174-y

40. A. Alloul, N.D. Christensen, C. Degrande, C. Duhr, B. Fuks, Comput. Phys. Commun. 185, 2250 (2014). https://doi.org/10.1016/j. cpc.2014.04.012

41. C. Degrande, C. Duhr, B. Fuks, D. Grellscheid, O. Mattelaer, T. Reiter, Comput. Phys. Commun. 183, 1201 (2012). https://doi.org/ 10.1016/j.cpc.2012.01.022

42. J. Alwall, R. Frederix, S. Frixione, V. Hirschi, F. Maltoni, O. Mattelaer, H.S. Shao, T. Stelzer, P. Torrielli, M. Zaro, JHEP 07, 079 (2014). https://doi.org/10.1007/JHEP07(2014)079

43. R.D. Ball, JHEP 04, 040 (2015). https://doi.org/10.1007/ JHEP04(2015)040

44. T. Sjöstrand, S. Ask, J.R. Christiansen, R. Corke, N. Desai, P. Ilten, S. Mrenna, S. Prestel, C.O. Rasmussen, P.Z. Skands, Comput. Phys. Commun. 191, 159 (2015). https://doi.org/10.1016/j.cpc.2015.01. 024

45. S. Alioli, P. Nason, C. Oleari, E. Re, JHEP 06, 043 (2010). https:// doi.org/10.1007/JHEP06(2010)043

46. L. Lönnblad, S. Prestel, JHEP 03, 019 (2012). https://doi.org/10. 1007/JHEP03(2012)019

47. M. Cacciari, G.P. Salam, G. Soyez, JHEP 04, 063 (2008). https:// doi.org/10.1088/1126-6708/2008/04/063

48. M. Cacciari, G.P. Salam, G. Soyez, Eur. Phys. J. C 72, 1896 (2012). https://doi.org/10.1140/epjc/s10052-012-1896-2

49. The ATLAS Experiment at the CERN Large Hadron Collider (2008). https://doi.org/10.1088/1748-0221/3/08/S08003

50. Expected Performance of the ATLAS Experiment-Detector, Trigger and Physics. CERN-OPEN-2008-020 (2009)

51. P. Pani, G. Polesello, Phys. Dark Univ. 21, 8 (2018). https://doi. org/10.1016/j.dark.2018.04.006

52. Expected performance of the ATLAS $b$-tagging algorithms in Run2. ATL-PHYS-PUB-2015-022 (2015). https://cds.cern.ch/record/ 2037697 
53. Optimisation and performance studies of the ATLAS $b$-tagging algorithms for the 2017-18 LHC run. ATL-PHYS-PUB-2017-013 (2017). https://cds.cern.ch/record/2273281

54. J. de Favereau, C. Delaere, P. Demin, A. Giammanco, V. Lemaître, A. Mertens, M. Selvaggi, JHEP 02, 057 (2014). https://doi.org/10. 1007/JHEP02(2014)057

55. P. Konar, K. Kong, K.T. Matchev, M. Park, JHEP 04, 086 (2010). https://doi.org/10.1007/JHEP04(2010)086
56. C.G. Lester, B. Nachman, JHEP 03, 100 (2015). https://doi.org/10. 1007/JHEP03(2015)100

57. A.L. Read, J. Phys. G 28, 2693 (2002). https://doi.org/10.1088/ 0954-3899/28/10/313. [11 (2002)]

58. L. Moneta, K. Belasco, K.S. Cranmer, S. Kreiss, A. Lazzaro, D. Piparo, G. Schott, W. Verkerke, M. Wolf, PoS ACAT2010, 057 (2010) 